\title{
Interference aware resource allocation model for D2D under cellular network
}

\author{
Ruksar Fatima, Rohina Khanam, Shaik Humera Tauseef \\ Khaja Bandanawaz College of engineering, Gulbarga, India
}

\begin{tabular}{l}
\hline \hline Article Info \\
\hline Article history: \\
Received Mar 11, 2019 \\
Revised Oct 17, 2019 \\
Accepted Oct 27, 2019 \\
\hline
\end{tabular}

\section{Keywords:}

Cellular network

D2D communication

Game theory

Interfernce management

Power allocation

Resource allocation

\begin{abstract}
Device-to-Device communication (D2D) has emerged as an efficient communication model in future generation cellular network for offloading cellular traffic and enhance overall network performance. D2D communication aid in attaining better spectrum utilization, lower delay, and less energy consumption, which can well adapt to meet demand of higher transmission rate, larger network capacity. Further, enhances spectral efficiency by reutilizing resource. However, it may result in severe cross-tier interference and co-tier interference. Therefore, efficient interference modelling design are required to address performance degradation caused by the interferences. The existing model has focused on addressing interference considering D2D association operating on same cell with the cellular association. As a result, it incurs interference to the cellular user located in the same cell. However, practically D2D association in overlapping area will reutilize spectrum of multiple neighboring cells. As a result, it incurs interference in multiple cells. For overcoming research challenges, this work presented Interference Aware Resource Allocation (IARA) model for D2D under cellular network as a game theory model. This work consider a resource allocation game where base station as a contender for catering D2D resource needs under different assumptions. Experiment are conducted to evaluate performance of IARA. The outcome shows IARA attained significant performance improvement over state-of-art models in terms of sum rate (utility), successful packet transmission, revenue, and delay.
\end{abstract}

Copyright $\odot 2020$ Institute of Advanced Engineering and Science. All rights reserved.

\section{Corresponding Author:}

Rohina Khanam,

Khaja Bandanawaz College of Engineering,

Gulbarga, India.

Email: rohina1119@rediffmail.com

\section{INTRODUCTION}

With the rapid growth of smart and wireless device has resulted in increased traffic at an annual rate of 53\% until 2020 [1]. As a result, the need for spectrum in cellular network is expected to go up exponentially in near future [2]. However, attaining high throughput within cellular network possess two challenges. Firstly, it is difficult to increase the physical layer capacity of wireless channel. Since physical layer technologies used in MIMO-OFDM ((Multiple Input Multiple Output-Orthogonal Frequency Division Multiplexing) and 4G/LTE (Long Term Evolution) with interference coordination and capacity-achieving codes, have reached their theoretical limits. Secondly, reducing the size of cell by using femtocell network is a achievable, yet costly, design for enhancing throughput, as it induces higher cost for offering backhaul connectivity of these small base stations.

Device-to-Device (D2D) communication has emerged has a new paradigm across academic and industries. This is mainly due to unique advantages it offers, the cellular network can offload its traffic, attain higher energy proficiency, better throughput, and robustness to infrastructure failures. Recently, AllJoyn, Qualcomm has aimed at addressing many challenges for enabling D2D communication. With the new 
Message Queue Telemetry Transport (MQTT) protocol is specifically designed only for device-to-device communication, the industry is well placed to its real-world deployment. The exiting model on D2D association [3] has still mainly focusing on how device-to-device communication can run proficiently as an underlay to cellular networks. Further, D2D is considered to be one of the promising technique in 5G communications. Here by sharing downlink or uplink resource blocks with cellular subscribers, device-to-device association attains a great potential of increasing network capacity and enhancing spectral efficiency [2]. D2D communication allows direct association among two subscribers which are geographically close to one another without being communicated by based station. This aid in attaining better spectrum utilization, lower delay, and less energy consumption, which can well adapt meet demand of higher transmission rate and larger network capacity $[4,5]$. Although enhanced spectral efficiency can be attained by the reutilization of resources in device-device associations. However, it may result in severe cross-tier interference (among cellular subscribers and D2D links) and co-tier interference (among different D2D links transmitting on the same resource blocks) [6,7]. If the interferences problem are not addressed properly, the performance of entire the network will be affected. Therefore, efficient interference modelling design are required to address quality-of service $(\mathrm{QoS})$ degradation caused by the interferences.

Recently, number of approaches is been presented to solve interference issues in device-to-device association underlying cellular networks [8-11]. In [8], presented guard zone based interference modelling for providing QoS requirement of cellular user. For mitigating interference due to presence of D2D association, [9] pairing based interference coordinating model. Further, [10] transmission capacity region which aid in managing interference in D2D environment when both successive interference cancellation and power control were carried out. Limiting co-existence of D2D pairs and cellular user imposed serious interference to the cellular network affecting overall capacity, in [11] considered both uplink and downlink interferences through admission control and power allocation, which intended at maximizing the overall throughput of the D2D associations underlying cellular network.

D2D association shares spectrum resources will result in challenges in co-existing of cellular and D2D communication due to joint interference. The existing model has focused on addressing interference considering D2D association is operating on same cell with the cellular association. As a result, it incurs interference to the cellular user located in the same cell (i.e. intra cell interference). However, practically D2D association will reutilize spectrum of multiple neighboring cells. As a result, it incurs interference in multiple cells (i.e., inter cell interference). This work aimed to addressing the inter cell interference of cellular network with presence of D2D. For example, when D2D user uses downlink spectrum resources of cellular network, a D2D user (Sender) may induce interference to a cellular user in the adjacent cell obtaining downlink load utilizing the same resource. Similarly, when a D2D users use uplink spectrum, the D2D user (Receiver) may incurs interference from a cellular subscriber in the adjacent cell transmitting uplink load to its associated base station. For overcoming (reduce) the interference among D2D and cellular subscribers, number of resource management approaches has been presented [12, 13]. However, most of the state-of-art resource allocation model has focused on addressing interference on the intra cell. However, inter cell scenario has be neglected.

In state-of-art D2D resource allocation model, game theory is widely used to obtain characteristics feature of competition between D2D associations. Game theory [14, 15] offers a mathematical solution for effective decision making, which offers a method to predict what may (or would) arise when rational contenders with varying interest. In state-of-art model $[16,17]$ D2D pairs is modelled as contender looking for the resources, where the utility of each contender is described as a function of generated interference and realizable data rate. The optimal solution (i.e., equilibrium) for resource allocation can be obtained by using utility function (i.e., contender's best response function). The state-of-art interference model [18] can be used to address inter cell condition by using interference information from adjacent cells. The operates of cellular network on the licensed band and it is very eminent to ensure the D2D radios that do not ensure the cellularusers. Same issues of the interference management can be encountered in the cognitive radio [19, 20], where the cellular usage is assumed main service. This can be prove highly benefical for network on the basis of cell-capacity [21]. However, it is not efficient when D2D tries to contend with the mutually shared channel of two adjacent cells and it also fails to determine resource configuration. Let consider an assumption where Device 1 and Device 2, are dispersed but proximal in the overlapping area of two adjacent cells. Device 1 and Device 2 must be in position of obtaining direct access as long as they are utilizing the mutual shared spectrum of two cells. As a result, there is need to remodel the game model to provision such condition.

For overcoming the research issues, this work present efficient resource allocation method in intercell D2D association underlying cellular network. Unlike state-of-art resource allocation model, this work considers a scenario where device-to-device link is positioned in the overlapping segment of adjacent cells. Very, limited work is carried to address the resource allocation issues for a D2D link positioned in overlapping (mutual) area of adjacent cells. Further, this considers base stations as a contender 
looking for resource allocation of D2D requested load rather than D2D pair as a contender like state-of-art model. Then, it defines the utility of each contender as the payoff obtained from both device-to-device and cellular association utilizing the resources. Further, we evaluate the existence of Nash Equilibrium properties. Thus, the payoffs of base stations can be improved.

The research contribution as follows

a. This work considers a case where device-to-device link is positioned in the overlapping segment of adjacent cells.

b. Further, presented a game theory model for resource allocation where base station is considered as contender for contending D2D pairs demand.

c. The Interference aware resource allocation model attain higher sum rate, successful packet transmission, revenue and less delay.

The rest of the paper is organized as follows. In section, 2 the proposed interference aware resource allocation model for D2D under cellular network is presented. In penultimate section, experimental study is carried out. The conclusion and future work is described in last section.

\section{INTERFERENCE AWARE RESOURCE ALLOCATION MODEL FOR D2D UNDER CELLULAR NETWORK}

This work present interference aware resource allocation model for D2D enabled under future high speed and crowded cellular network. Firstly, this briefly discusses about interference model and architecture considered for resource allocation. Then, this model present a game theory model that maximize the sum rate (utility) of resource allocation considering different assumption. The architecture of interference assumption considered in this work is presented in Figures 1-3.

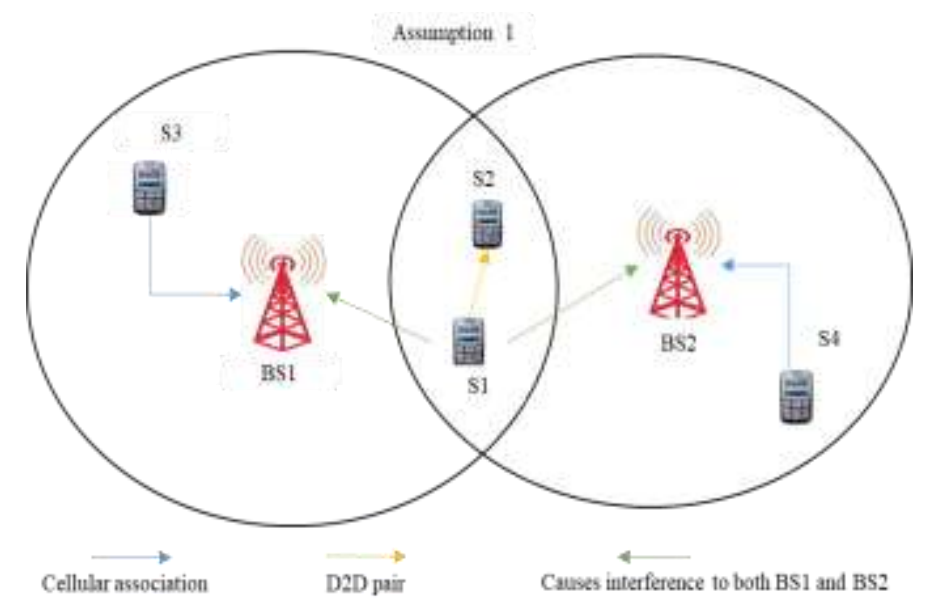

Figure 1. Architecture of assumption 1 of D2D communication under cellular network

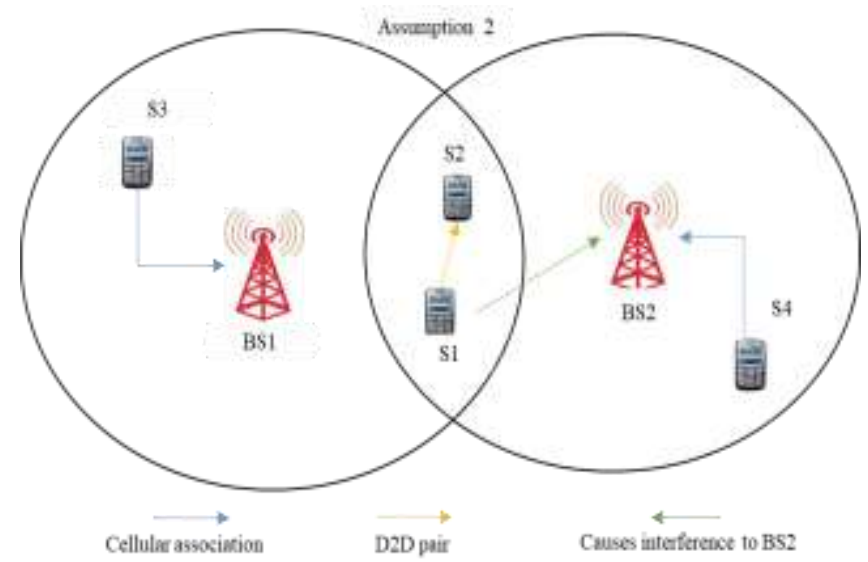

Figure 2. Architecture of assumption 2 of D2D communication under cellular network 


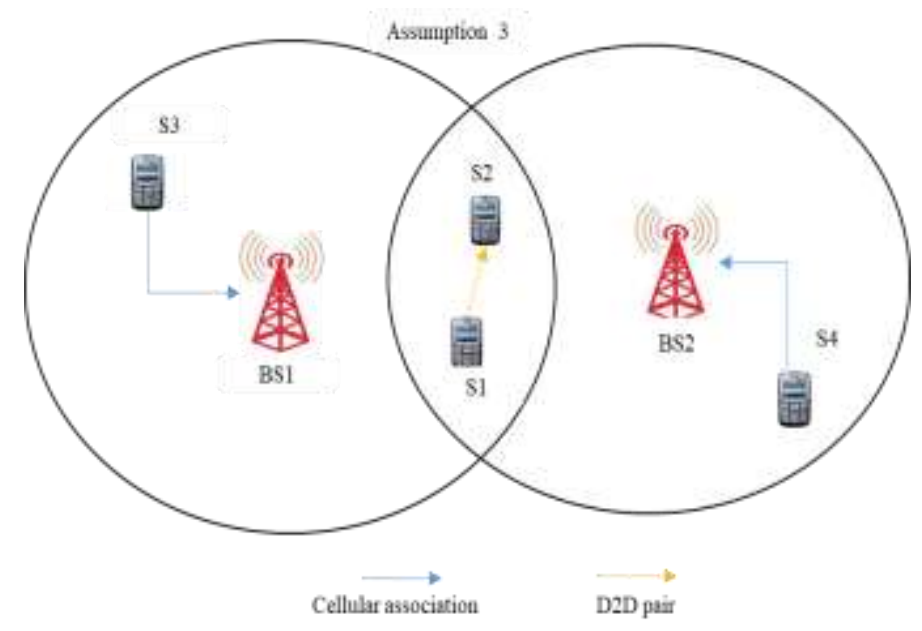

Figure 3. Architecture of assumption 3 of D2D communication under cellular network

\section{a) System model for D2D network:}

This section describes the D2D system model considered for efficient resource allocation among contending D2D subscribers. This work considers a case where two D2D subscribers are positioned in the overlying region of two adjacent cells using uplink resource for association. In each cell, there exits at least one cellular subscriber that associates with base station. This work further considers that D2D pair reutilize the uplink resources among cellular subscribers while cellular associations use either downlink or uplink resource, thus aid in synchronizing with base stations. The case can be expressed in to following three assumption, where subscriber 1 (source) and subscriber 2 (destination) are the D2D pair, subscriber 3 and subscriber 4 are the cellular subscriber.

- Assumption 1: Cellular associations (blue line) in both cells utilize uplink resources. Here, D2D pair (yellow line) induces interference (red line) among base stations as shown in Figure 1.

- Assumption 2: Cellular associations in the right side cell utilize uplink resource while in the left side utilize the downlink resource. In this assumption, D2D associations only induces interference to base station 2 as shown in Figure 2.

- Assumption 3: Cellular association in both cell utilize downlink resource. In this case, D2D pair induces no interference among base stations as shown in Figure 3. Let depict the rate allocation of base station of above assumptions. Let $H_{j k}$ be the channel power gain among source $j$ and destination $k$ over a D2D channel or cellular channel, $H_{3 B_{1}}$ be the gain among subscriber 1 and base station $1, H_{3 B_{2}}$ be the gain among subscriber 1 and base station 2. The source $j$ power is denoted by $q_{j}$, the rate allocation of $n^{\text {th }}$ base station in assumption $o$ by $S_{n}^{o}$, the additive white Gaussian noise power at the destination is represented by $O_{0}$. Subsequently, for assumption 1 , we obtains

$$
\begin{aligned}
& S_{1}^{1}=C_{1} * \log _{2}\left(1+\frac{H_{1 B_{1} q_{1}}}{O_{0}+H_{3 B_{1} q_{3}}}\right), \\
& S_{2}^{1}=C_{2} * \log _{2}\left(1+\frac{H_{2 B_{2} q_{2}}}{O_{0}+H_{3 B_{2} q_{3}}}\right) .
\end{aligned}
$$

where $S_{1}$ and $S_{2}$ are the data rate of base station 1 and base station 2, respectively.

Further, for assumption 2, we obtain

$$
\begin{aligned}
& S_{1}^{2}=C_{1} * \log _{2}\left(1+\frac{H_{B_{1} 1 q B_{1}}}{O_{0}}\right), \\
& S_{2}^{2}=C_{2} * \log _{2}\left(1+\frac{H_{2 B_{2} q_{2}}}{O_{0}+H_{3 B_{2} q_{3}}}\right) .
\end{aligned}
$$


Similarly, for assumption 3 , we obtain

$$
\begin{aligned}
& S_{1}^{3}=C_{1} * \log _{2}\left(1+\frac{H_{B_{1} 1 q B_{1}}}{O_{0}}\right), \\
& S_{2}^{1}=C_{2} * \log _{2}\left(1+\frac{H_{B_{1} 2 q B_{2}}}{O_{0}}\right) .
\end{aligned}
$$

The rate allocation of all base stations aid in obtaining their revenue attained from cellular associations. An important thig to be seen here is that the model to evaluate rate allocation considers interference induced among D2D channel, which aid in impacting revenue of the base station. For example, allocating more bandwidth resource to D2D user will result in reduced interference. As a result, careful planning is required in allocating resource to D2D subscriber in future high speed mobile network. This work present a game theory approach to estimate the amount of bandwidth to be assigned to D2D source subscriber by respective base stations. This work considers that the base station has all information regarding channel state information of each channel in the available cell with respect to base stations. So that overlapping base station can synchronize the radio resource.

\section{b) Resource allocation using game theory approach for D2D network:}

This section describes the resource allocation using game theory approach. Let considers that the each service provider can charge its D2D subscriber. Since D2D channel reutilize the shared channel among two cell. Thus, service providers can maximize their revenue and possess incentive to assign resource to D2D subscriber. The race among service provider can be considered as cooperative game, in which base stations are modelled as contenders. This section present a game theory model where each base station can obtain information of policies and revenue of the base station. Let considers the utility of contender as the regulatory payoffs that composed of two phases. The first phase is obtained from charges obtained from cellular subscribers, and second phase is the charges obtained from D2D association. Therefore, the utility parameter of contenders are expressed as follows

$$
\begin{aligned}
& V_{1}^{o}=\varphi * S_{1}^{o}+\omega * C_{3}^{\tau}-\mu * C_{3}^{\tau}, \\
& V_{2}^{o}=\varphi * S_{2}^{o}+\omega * C_{3}^{\rho}-\mu * C_{3}^{\rho},
\end{aligned}
$$

where $\varphi$ and $\omega$ are constant depicting the subscription charges of unite bandwidth and resource, respectively. The allocated bandwidth from base station 1 and base station 2 is represented by $C_{3}^{\tau}$ and $C_{3}^{\rho}$ respectively and $\mu$ is the cost objective policies of resource. The pricing policies with respect to $\mu$ is used similar to [22, 23]. Therefore, it can be formulated as follows

$$
\mu=y+z\left(C_{3}^{\tau}+C_{3}^{\rho}\right)^{\alpha}
$$

where $y, z$, and $\alpha$ are constant with non-negative parameter, and $\alpha \geq 1$ assures that the cost objective policies is convex. The (9), depicts that the cost of all base stations is directly proportional to resource attained by D2D subscriber. Let consider a base $\operatorname{station} B_{2}$, maximize its utility by individually increase the resource allocation, the cost of all base stations would be optimized accordingly. This result, in reducing the utility of base station $B_{1}$, and thus base station 1 has incentive to assign more resource competing with base station 2. As there exist trade-off among data rate and power [24], which can be obtained as follows

$$
q_{3} \propto \frac{1}{C_{3}^{\tau}+C_{3}^{\rho}}
$$

For easiness, we consider

$$
\left[O_{0}+H_{3 B_{1}} q_{3}\right]=\frac{a_{1}}{C_{3}^{\tau}}
$$




$$
\left[O_{0}+H_{3 B_{2}} q_{3}\right]=\frac{a_{2}}{C_{3}^{\rho}}
$$

where $a_{1}$ and $a_{2}$ are constant with non-negative parameter, thus we have

$$
\begin{aligned}
& V_{1}^{o}=\varphi * S_{1}^{o}+\omega * C_{3}^{\tau}-\left[y+z\left(C_{3}^{\tau}+C_{3}^{\rho}\right)^{\alpha}\right] * C_{3}^{\tau}, \\
& V_{2}^{o}=\varphi * S_{2}^{o}+\omega * C_{3}^{\rho}-\left[y+z\left(C_{3}^{\tau}+C_{3}^{\rho}\right)^{\alpha}\right] * C_{3}^{\rho},
\end{aligned}
$$

The optimization strategy for obtaining efficient resource allocation for D2D association is expressed as follows

$$
\begin{aligned}
& \max V_{n}^{o}\left(C_{3}^{\tau}+C_{3}^{\rho}\right) \\
& \text { such that } C_{\downarrow} \leq C_{3}^{\tau}+C_{3}^{\rho} \leq C_{\uparrow}, \\
& 0 \leq C_{3}^{\tau} \leq C_{1}, \\
& 0 \leq C_{3}^{\rho} \leq C_{2}, \\
& \varphi, \omega, y, z>0, \\
& a_{1}, a_{2} \geq 0, \\
& \alpha \geq 1 .
\end{aligned}
$$

where $C_{\downarrow}$ and $C_{\uparrow}$ are the minimum and maximum bandwidth requirement of D2D pair. An important things to be seen here is that $C_{\downarrow}$ is utilized to control implicitly the D2D communication power that doesn't incurs much interference among base stations and the cellular subscribers. Similarly, the D2D channel reutilizes the shared resources of two cells, the resource allocated to D2D by these base stations must not surpass to the allocated bandwidth with respect to cellular subscriber in each cell, thus, we obtain $0 \leq C_{3}^{\tau} \leq C_{1}$ and $0 \leq C_{3}^{\rho} \leq C_{2}$. By considering Nash equilibrium, we can attain efficient resource allocation by solving the ideal strategy policies of each contender which is expressed as follows

$$
\begin{aligned}
& \left(\hat{C}_{3}^{\tau}, \hat{C}_{3}^{\rho}\right)=\arg \max V_{n}^{o}\left(C_{3}^{\tau}, C_{3}^{\rho}\right) \\
& \text { such that } C_{\downarrow} \leq C_{3}^{\tau}+C_{3}^{\rho} \leq C_{\uparrow}, \\
& 0 \leq C_{3}^{\tau} \leq C_{1}, \\
& 0 \leq C_{3}^{\rho} \leq C_{2}, \\
& \varphi, \omega, y, z>0 \\
& a_{1}, a_{2} \geq 0 \\
& \alpha \geq 1 .
\end{aligned}
$$$$
\text { such that } C_{\downarrow} \leq C_{3}^{\tau}+C_{3}^{\rho} \leq C_{\uparrow} \text {, }
$$

Further, to attain Nash equilibrium of (13) fixed game, we differentiate $V_{n}^{o}$ with respect to $C_{3}^{\tau}$ and $C_{3}^{\rho}$, respectively. Thus, we have

$$
\left\{\begin{array}{c}
\frac{\partial V_{1}^{o}\left(C_{3}^{\tau}, C_{3}^{\rho}\right)}{\partial V_{3}^{\tau}}=0 \\
\frac{\partial V_{2}^{o}\left(C_{3}^{\tau}, C_{3}^{\rho}\right)}{\partial V_{3}^{\rho}}=0 \\
0 \leq C_{3}^{\tau} \leq C_{1} \\
0 \leq C_{3}^{\rho} \leq C_{1} \\
C_{\downarrow} \leq C_{3}^{\tau} \leq C_{3}^{\rho} \leq C_{\uparrow}
\end{array}\right.
$$

The proposed resource allocation model attains significant result which is experimentally proved in next section. 


\section{SIMULATION RESULTS AND ANALYSIS}

This section present experiment evaluation of proposed interference aware resource allocation (IARA) model for D2D pair under cellular network over state-of-art models. The performance of IARA model is evaluated over existing models in terms of Sum rate, packet communication delay, successful packet transmission, and cost incurred to perform data transmission. This work consider a cell scenario, where D2D pairs and cellular users are uniform randomly distributed in a square area of $1000 \mathrm{~m} \times 1000 \mathrm{~m}$ with the base station in the center. Further, the D2D pairs and cellular subscribers are mobile in nature with uniform speed. For a fixed number of D2D pairs and cellular subscribers, we repeat the simulation by 2 times and mean of the result is taken. The simulation parameter considered for this work is similar to [17], and [18]. More detail of simulation parameter used for simulation study is described in Table 1.

Table 1. Simulation parameter considered

\begin{tabular}{ll}
\hline Network Parameter & Value \\
\hline Number of cell & 16 \\
Cell width & $1000 \mathrm{~m}$ \\
Number of cellular and D2D users & 200 \\
Modulation scheme & QPSK \\
Mobility of devices & $5 \mathrm{~m} / \mathrm{s}$ \\
Maximum distance of D2D & $100 \mathrm{~m}$ \\
Sub-carrier bandwidth & $1 \mathrm{~Hz}(15 \mathrm{kHz})$ \\
Time slot duration & $5 \mu \mathrm{s}$ \\
Number of Frequency Channels & $10,20,30$, and 40 \\
Path loss exponent & 3.5 \\
\hline
\end{tabular}

\section{a) Sum rate (utility) performance evaluation}

This section evaluates sum rate as a utility performance of IARA over existing model [3, 16, 17]. For experiment analysis we have considered following assumption such as, cellular associations in both (adjacent/neighbouring) cells utilize uplink resources. Here, D2D pair induces interference among base stations as shown in Figure 1. The sum rate performance is evaluated by varying link/channel size and the outcome attained by IARA and exiting model is graphically shown in Figure 4. The outcome shows that IARA improves sum rate performance by $4.19 \%, 34.87 \%, 8.19 \%$, and $5.5 \%$ over exiting model considering varied channel size of $10,20,30$, and 40, respectively. An average sum rate performance improvement of $13.47 \%$ is attained by IARA over existing model. Similarly, experiment are conducted considering following assumption such as, cellular associations in the one cell utilize uplink resource while in the other cell utilize the downlink resource. In this assumption, D2D associations only induces interference to base station 2 as shown in Figure 2. The sum rate performance is evaluated by varying link/channel size and the outcome attained by IARA and exiting model is graphically shown in Figure 5. The outcome shows that IARA improves sum rate performance by $6.05 \%, 12.8 \%, 10.07 \%$, and $9.42 \%$ over exiting model considering varied channel size of 10,20,30, and 40, respectively. An average sum rate performance improvement of $9.61 \%$ is attained by IARA over existing model. Further, experiment are conducted considering following assumption such as, cellular association in both cell utilize downlink resource. In this case, D2D pair induces no interference among base stations as shown in Figure 3. The sum rate performance is evaluated by varying link/channel size and the outcome attained by IARA and exiting model is graphically shown in Figure 6. The outcome shows that IARA improves sum rate performance by $6.08 \%, 15.87 \%, 8.35 \%$, and $11.29 \%$ over exiting model considering varied channel size of 10, 20,30, and 40, respectively. An average sum rate performance improvement of $10.49 \%$ is attained by IARA over existing model. The overall result attained shows an average sum rate improvement of $11.19 \%$ is achieved by IARA over existing model. Further, no prior work as considered such performance evaluation assumption. Hence, the proposed model is scalable and robust.

\section{b) Successful packet transmission performance evaluation}

This section evaluates successful packet transmission performance of IARA over existing model [3, 16, 17]. For experiment analysis we have considered all three assumption of Figure 1, Figure 2, Figure 3 together and simulation is conducted. The successful packet transmission performance is evaluated by varying link/channel size and the outcome attained by IARA and exiting model is graphically shown in Figure 7. The outcome shows that IARA improves packet transmission performance by $4.87 \%, 37.18 \%$, $9.32 \%$, and $5.67 \%$ over exiting model considering varied channel size of 10, 20, 30, and 40, respectively. An average successful packet transmission performance improvement of $14.76 \%$ is attained by IARA over existing model. The overall result attained by IARA is scalable and robust. 


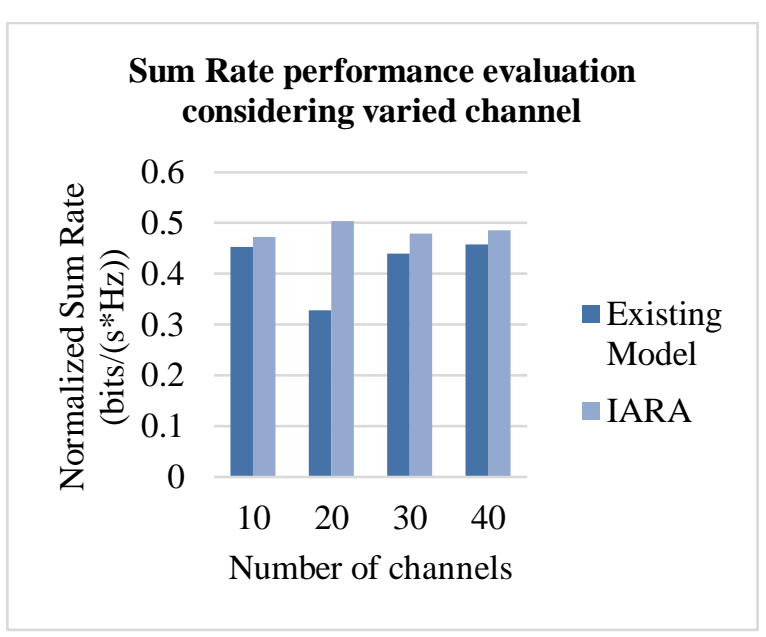

Figure 4. Sum rate performance evaluation considering varied channel under assumption 1 (Figure 1)

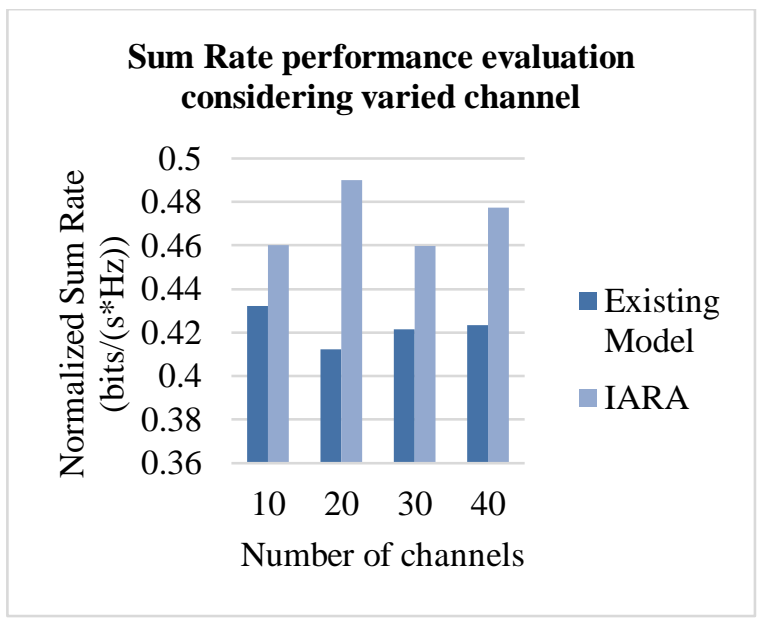

Figure 6. Sum rate performance evaluation considering varied channel under assumption 3

(Figure 3)

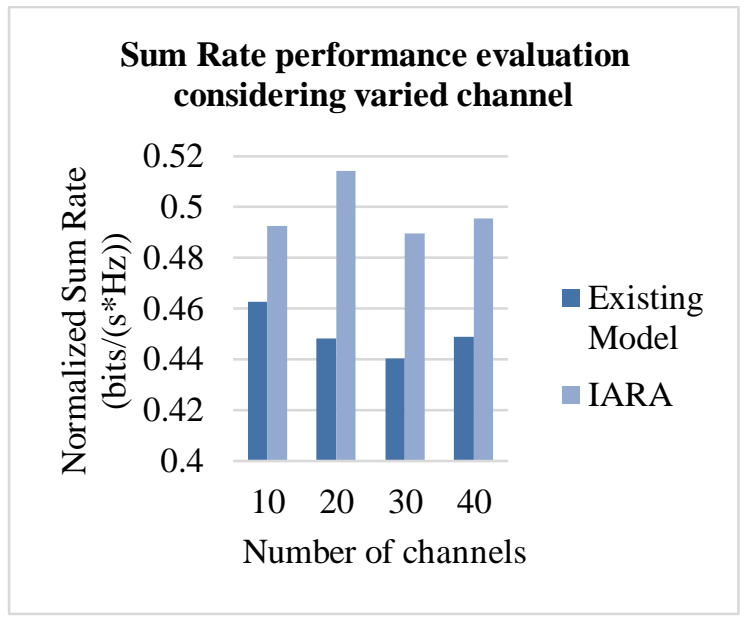

Figure 5. Sum rate performance evaluation considering varied channel under assumption 2

(Figure 2)

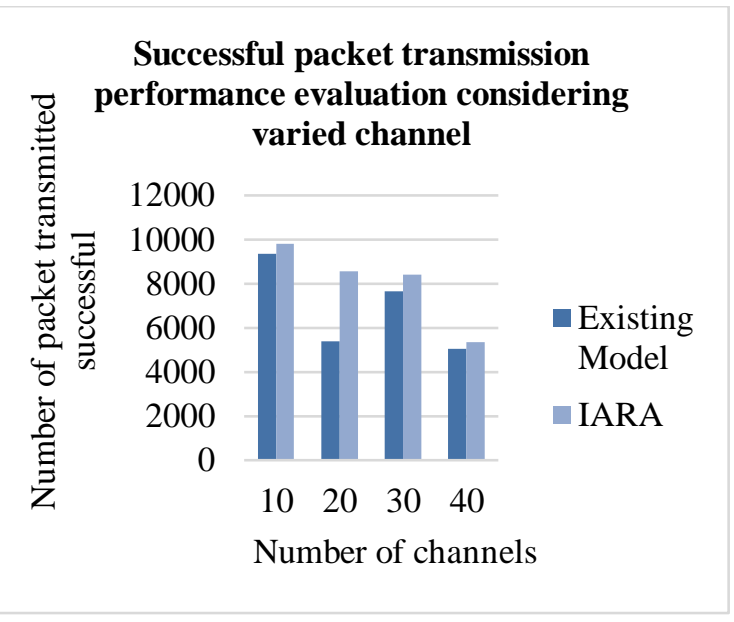

Figure 7. Successful packet transmission performance evaluation considering varied channel

c) Delay performance evaluation:

This section evaluates delay performance of IARA over existing model [3, 16, 17]. The delay is computed in terms of the number of packets being dropped with respect to overall packet being transmitted [25]. For experiment analysis we have considered all three assumption of Figure 1, Figure 2, Figure 3 together and simulation is conducted. The delay performance is evaluated by varying link/channel size and the outcome attained by IARA and exiting model is graphically shown in Figure 8 . The outcome shows that IARA reduces delay by $3.62 \%, 26.17 \%, 7.02 \%$, and $4.93 \%$ over exiting model considering varied channel size of 10, 20,30, and 40, respectively. An average delay reduction of $11.27 \%$ is attained by IARA over existing model. The overall result attained by IARA is scalable and robust.

\section{d) Revenue performance evaluation}

This section evaluates revenue performance of IARA over existing model $[3,16,17]$. The revenue is computed similar to [13]. For experiment analysis we have considered all three assumption of Figure 1, Figure 2, Figure 3 together and simulation is conducted. The revenue performance is evaluated by varying link/channel size and the outcome attained by IARA and exiting model is graphically shown in Figure 9. The outcome shows that IARA increase revenue by $30.34 \%, 4.63 \%, 27.3 \%$, and $29.67 \%$ over exiting model considering varied channel size of 10, 20, 30, and 40, respectively. An average revenue improvement of $23.5 \%$ is attained by IARA over existing model. The overall result attained by IARA is scalable and robust. 


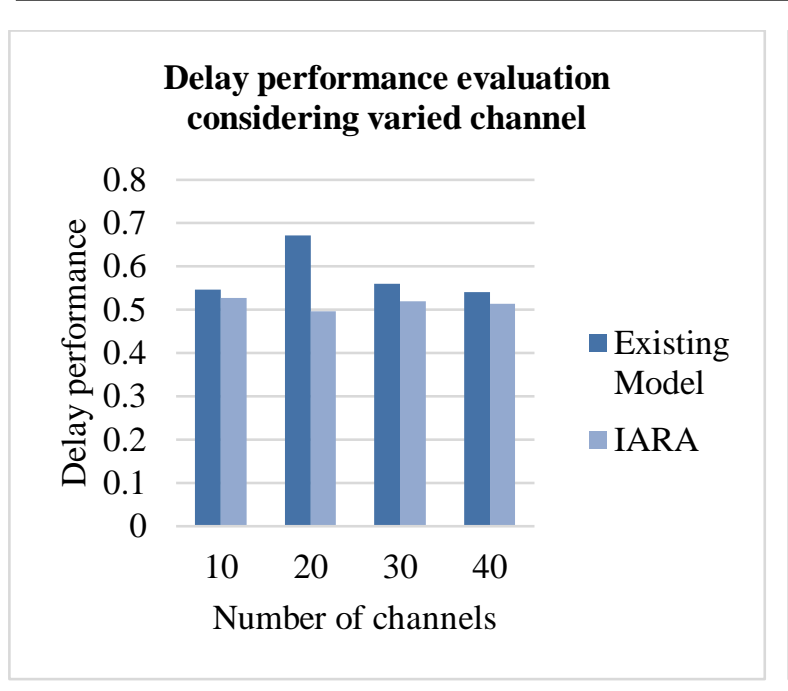

Figure 8. Delay performance evaluation considering varied channel

\section{Revenue performance evaluation considering varied channel}

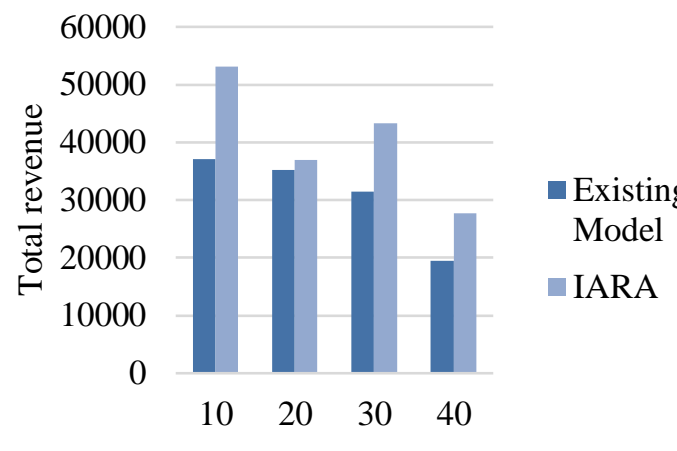

Number of channels

Figure 9. Revenue performance evaluation considering varied channel

\section{e) Result and discussion}

Number of model has been presented recently for addressing resource allocation problem for D2D pair under cellular network. In [3] aimed at maximizing cellular traffic offloading to D2D by adopting caching mechanism. Experiment are conducted to evaluate outcome in term of performance gain and results shows good performance. However, it is affected due to interference to cellular and induce delay. Since it required network-wide information to obtain optimal solution. For addressing delay, [25] presented a content caching using matching algorithm. Experiment outcome shows reduction delay. However, their model considers only single base station and sum rate performance is not considered for evaluation. Further, number of approaches has been presented adopting game theory for resource allocation considering multiple base station $[13,18]$. Further, $[16,17]$ presented resource allocation model in overlapping section of two base station. The state-of-art model can be used to address inter cell condition by using interference information from adjacent cells. However, it is not efficient when D2D tries to contend with the mutually shared channel of two adjacent cells and it also fails to determine resource configuration. As a result, this work we presented a new game model for resource allocation for D2D pair considering different assumption and attained higher utility (sum rate), successful packet transmission, revenue ad less delay than state-of-art model.

\section{CONCLUSION}

D2D communication aid enhancing spectral efficiency by enabling resource reutilization. As resource (spectrum) is shared among user. As a result, it incurs interference among contending cellular user. Number of approaches has been presented to address interference issue in D2D enabled cellular network. However, the state-of-art resource allocation model are designed considering D2D association operating on same cell with the cellular association. As a result, it incurs interference to the cellular user located in the same cell. Further, in general D2D association in overlapping area will reutilize spectrum of multiple neighboring cells. As a result, it incurs interference in multiple cells. Thus affecting the system performance. This work presented an Interference Aware Resource Allocation model for D2D under cellular network. The resource allocation model is designed as a game theory approach where base station is considered as a contender for meeting resource need of D2D pair presented in overlapping area of adjacent cells. We modelled the resource allocation problem considering various assumption interference. Experiment are conducted to evaluate performance of IARA. The outcome shows IARA attain superior performance improvement over state-of-art models in terms of sum rate (utility), successful packet transmission, revenue, and delay. An average sum rate (utility) performance improvement of $11.55 \%$ is attained by IARA over existing models considering varied assumption. Further, an average successful packet transmission and revenue performance improvement of $14.76 \%$ and $23.5 \%$, respectively is attained by IARA over existing models considering varied assumption. Then, delay reduction of $11.27 \%$ is attained by IARA over existing model. The overall result attained shows scalable and robust performance. In future, this work would consider performance evaluation considering various other parameter. Further, we will enhance the game theory for attaining better resource allocation and enhance the sum rate. 


\section{REFERENCES}

[1] Cisco Virtual Networking Index (VNI) Forecast, "Cisco visual networking index: Global mobile data traffic forecast update, 2015-2020,” C. V. N. Index, Cisco, Tech. Rep.c11-520862, 2016. [Online]. Available: http://www.cisco.com/c/dam/m/en in/innovation/enterprise/assets/mobilewhite-paper-c11-520862.pdf.

[2] G. Mahalakshmi and V. M. Bhaskaran, "Managing mobility in wireless cellular networks: A profile based approach," 2014 International Conference on Electronics and Communication Systems (ICECS), Coimbatore, 2014, pp. 1-5.

[3] J. Jiang, S. Zhang, B. Li and B. Li, "Maximized Cellular Traffic Offloading via Device-to-Device R. Alkurd, R. M. Shubair, and I. Abualhaol, "Survey on device-todevice communications: Challenges and design issues," in 2014 IEEE 12th International New Circuits and Systems Conference (NEWCAS), Trois-Rivieres, QC, Canada, 2014, pp. 361-364.

[4] P. Mach, Z. Becvar, and T. Vanek, "In-band device-to-device communication in ofdma cellular networks: A survey and challenges," IEEE Communications Surveys Tutorials, vol. 17, no. 4, pp. 1885-1922, 2015.

[5] G. A. Safdar, M. Ur-Rehman, M. Muhammad, M. A. Imran, and R. Tafazolli, "Interference mitigation in d2d communication underlaying lte-a network," IEEE Access, vol. 4, pp. 7967-7987, 2016.

[6] Y. Hassan, F. Hussain, S. Hossen, S. Choudhury, and M. M. Alam, "Interference minimization in d2d communication underlaying cellular networks," IEEE Access, vol. 5, pp. 22 471-22 484, 2017.

[7] S. Lv, C. Xing, Z. Zhang, and K. Long, "Guard zone based interference management for d2d-aided underlaying cellular networks," IEEE Transactions on Vehicular Technology, vol. 66, no. 6, pp. 5467-5472, 2017.

[8] R. Yin, G. Yu, H. Zhang, Z. Zhang, and G. Y. Li, "Pricing-based interference coordination for d2d communications in cellular networks," IEEE Transactions on Wireless Communications, vol. 14, no. 3, pp. 1519-1532, 2015.

[9] M. Shen, J. Liu, X. Wang, Y. Zhang, H. Sun, and J. Li, "On transmission capacity region of d2d integrated cellular networks with interference management," IEEE Transactions on Communications, vol. 63, no. 4, pp. 1383-1399, 2015.

[10] T. Huynh, T. Onuma, K. Kuroda, M. Hasegawa, and W. J. Hwang, "Joint downlink and uplink interference management for device to device communication underlaying cellular networks," IEEE Access, vol. 4, pp. 4420-4430, 2016.

[11] Content Sharing," in IEEE Journal on Selected Areas in Communications, vol. 34, no. 1, pp. 82-91, Jan. 2016. doi: 10.1109/JSAC.2015.2452493.

[12] Z. Zhou, K. Ota, M. Dong and C. Xu, "Energy-Efficient Matching for Resource Allocation in D2D Enabled Cellular Networks," in IEEE Transactions on Vehicular Technology, vol. 66, no. 6, pp. 5256-5268, June 2017.

[13] Y. Liu, R. Wang and Z. Han, "Interference-Constrained Pricing for D2D Networks," in IEEE Transactions on Wireless Communications, vol. 16, no. 1, pp. 475-486, Jan. 2017.

[14] C. Gao, Y. Li, Y. Zhao and S. Chen, "A Two-Level Game Theory Approach for Joint Relay Selection and Resource Allocation in Network Coding Assisted D2D Communications," in IEEE Transactions on Mobile Computing, vol. 16, no. 10, pp. 2697-2711, 1 Oct. 2017.

[15] J. Huang, C. Xing, Y. Qian and Z. J. Haas, "Resource Allocation for Multicell Device-to-Device Communications Underlaying 5G Networks: A Game-Theoretic Mechanism With Incomplete Information," in IEEE Transactions on Vehicular Technology, vol. 67, no. 3, pp. 2557-2570, March 2018.

[16] J. Hu, W. Heng, Y. Zhu, G. Wang, X. Li and J. Wu, "Overlapping Coalition Formation Games for Joint Interference Management and Resource Allocation in D2D Communications," in IEEE Access, vol. 6, pp. 6341-6349, 2018.

[17] Y. Zhao, Y. Li, D. Wu and N. Ge, "Overlapping Coalition Formation Game for Resource Allocation in Network Coding Aided D2D Communications," in IEEE Transactions on Mobile Computing, vol. 16, no. 12, pp. 3459-3472, 1 Dec. 2017.

[18] Y. Chen, B. Ai, Y. Niu, K. Guan and Z. Han, "Resource Allocation for Device-to-Device Communications Underlaying Heterogeneous Cellular Networks Using Coalitional Games," in IEEE Transactions on Wireless Communications, vol. 17, no. 6, pp. 4163-4176, June 2018.

[19] S. Haykin, "Cognitive radio: Brain-empowered wireless communications," IEEE Journal on Selected Areas in Communications, vol. 23, no. 2, pp. 201-220, Feb. 2005.

[20] J. M. III and J. Gerald Q. Maguire, "Cognitive radio: Making software radios more personal," IEEE Personal Communications, vol. 6, no. 4, pp. 13-18, Aug. 1999.

[21] G. Mahalakshmi and V. M. Bhaskaran, "Managing mobility in wireless cellular networks: A profile based approach," 2014 International Conference on Electronics and Communication Systems (ICECS), Coimbatore, 2014, pp. $1-5$.

[22] Lingareddy, Sanjay \& Charles, B \& Babu, Vinaya \& Dhruve, Kashyap. (2009). Wireless Information Security Based on Cognitive Approaches.

[23] Ekram Hossain and Zhu Han, "Game theory for Cognitive Radio Networks", available: http:/globecom2009.ieeeglobecom.org/Uploads/Public/Docs_Globecom_2009/ekram-zhu-tutorial_14.pdf.

[24] B. P. Lathi and Z. Ding, Modern Digital and Analog Communication Systems. Oxford University Press, 2009.

[25] J. Li et al., "On Social-Aware Content Caching for D2D-Enabled Cellular Networks with Matching Theory," in IEEE Internet of Things Journal. doi: 10.1109/JIOT.2017.2749320, 2017. 